\title{
Effects of surfactant on the enzymatic degradation of oil palm empty fruit bunch
} (OPEFB)

\begin{abstract}
Various pretreatments have been employed to pretreat oil palm empty fruit bunch (OPEFB) to improve sugars production via enzymatic degradation reaction. However, less attention has been paid to investigate the effects of surfactant addition in the OPEFB degradation process. Therefore, the effects of surfactants on degradation of pretreated OPEFB by Aspergillus niger EFB1 crude cellulase were studied. Tween 80 was the most effective surfactant tested. When $0.25 \%(\mathrm{v} / \mathrm{v})$ Tween 80 was added, the production of reducing sugar increased $63 \%$ (from 0.49 to $0.8 \mathrm{~g} / \mathrm{L}$ ). This was the optimum yield recorded. It also improved cellobiose, glucose and xylose production by two folds, seven folds and one fold higher than that without Tween 80, respectively. Tween 80 reduced the loss of cellulases activities where more than $17 \%$ of the original cellulases activities were retained after 7 days of degradation. Nonetheless, Tween 80 did not improve the protein concentration consistently. Supplementation of Tween 80 increased lignin removal by $23 \%$. Observations using SEM revealed that, with Tween 80 , the presence of pores and surface cracks were more pronounced on the surface of degraded OPEFB fibers. As a whole, the reported effects showed an improved production of reducing sugars in the presence of Tween 80 . Thus, Tween 80 addition appeared as a promising method in enhancing the bioconversion of OPEFB into value-added co-products.
\end{abstract}

Keyword: Tween 80; Biodegradation; Cellulose; Lignin; Sugar; Cellulase activity 